\title{
Enhancement of Radio Link of Cellular Cell using multiple antennas
}

\author{
Karar khaleel aljawaheri ${ }^{1}$, Neelesh Agrawal ${ }^{2}$, A. K. Jaiswal ${ }^{3}$, Novendu Nitin ${ }^{2}$ \\ ${ }^{1}$ (Master of Technology student, Department of Electronic and Communication/Wireless Communication \\ Engineering, Technical College - Najaf, Iraq) \\ 2 (Assistant Professor, Department of Electronic and Communication, SHIAT, India) \\ ${ }^{3}$ (Professor, Department of Electronic and Communication, SHIAT, India)
}

\begin{abstract}
The mobile radio channel puts various limitations on the performance of wireless or mobile communications system. In wireless communications, the transmission path between the transmitter and receiver can vary from simple line-of-sight to one that is severely obstructed by buildings, mountains and foliage so high data rate services are highly required. In this paper, Distributed antenna system (DAS) is combined with the diversity characteristics in order to enhance the mobile radio channel also to get high data rate. Distributed antenna system (DAS) are traditionally deployed for the coverage purpose by replacing the single antenna of the traditionally cellular system with multiple antennas geographically separated within the cell and connected back to the main base station. Diversity is a powerful communication receiver technique that provides wireless link improvement at relatively low cost. The downlink scenarios for DAS with single antenna selection (SAS) and multiple antenna selection (MAS) have been reviewed also diversity technique which is applied through MIMO system is analyzed. According to the system level simulation, the cell signal to interference plus noise ratio, cell edge throughput and capacity of the system is improved by $35 \%, 82 \%$ and $75 \%$ respectively. Moreover, the total transmitted power can be reduced by $75 \%$.
\end{abstract}

Keywords: Distributed Antenna System (DAS), Diversity Technique, Multiple antennas

\section{INTRODUCTION}

Mobile communications is one of the greatest commercial success stories of the last decade of the previous millennium. Mobile communications is not a new phenomenon; its success started from the time cellular systems were introduced in 1980s. Over the past two decades, the cellular mobile communication industry has continued to record an amazing growth, with the number of world-wide users expected to pass the three billions mark soon. However, all is not well in the mobile communications world. The main issue is that an increase in the number of users also means more capacity with excellent coverage will be required. To capture all this huge market, the industry must develop new technologies to increase overall capacity, and to reduce the drop-call rate, which affects the quality of service of individual networks, especially in new applications requiring very high-data rates. This is not an easy task, considering all the restrictions on designers of mobile communication systems [1]. A distributed antenna system (DAS) is a network of spatially distributed antenna elements (DAE) that are connected to a common source via a wired transport medium, usually optical fiber, to provide wireless service within geographic area. DASs were originally introduced to simply cover the dead spots in indoor communication; further studies have identified other potential advantage such as power saving, system capacity and enhance the coverage. Later, research of DASs has taken two main lines; the first line is to model DASs as variation of conventional MIMO system where the antenna elements are geographically distributed. However, in general, a DAS is different from a MIMO system in the following points:

1. In a DAS, each DAE has different channel gain since uplink/downlink signals between the user terminals and the different antenna elements experience different multipath, shadowing and path loss characteristics.

2. In a DAS, each DAE has its own power unlike MIMO system; a common power constraint is imposed.

3. The dedicated links used to connect the DAEs to the central processing unit might be subject to delay or other constraints.

Due to these differences, channel modeling and performance analysis of DASs may not be carried out using existing tools for conventional MIMO and different mathematical tools needed. The other line of research is based on using MIMO has been used within each DAE to reduce the small scale fading might happen at location within the cell [13].

\section{Multiple Antenna System}

The propagation problems of the radio waves have the direct effect on the cellular system performance so now it is necessary to think how it can be decreased as much as possible, at the same time the demand for higher 
data rates at better service quality is challenged by the scarce usable radio resources as well as time-varying radio environment, which are both affected by fading and multipath. In this scenario, the technique of using multiple antennas has been widely accepted as one that clearly improves the data rate on longer ranges without consuming extra bandwidth or transmission power and this solution consider non costly.

In this paper, two approaches of using multiple antennas are presented; one approach is to enhance the quality of signals at the cell border. This is implemented by spatially distributing antennas within the cell of the cellular system. This technique is referred to as the Distribution Antenna System (DAS) [5, 6].

The second approach is to decrease the small-scale or deep fading; it means more reliability that can be offered by each sector of the cellular cell. This is done by using Multiple-Input Single-Output (MISO) systems which exploits diversity at the transmitter through the use of $M_{T}$ transmits antennas in combination with preprocessing or precoding (Space-Time Coding). A significant difference with receive diversity is that the transmitter might not have the knowledge of the MISO channel. Indeed, at the receiver, the channel is easily estimated. This is not the case at the transmit side, where feedback from the receiver is required to inform the transmitter. However, there are basically two different ways of achieving direct transmit diversity [14],

1. When the transmitter has a perfect channel knowledge, MIMO beam-forming can be performed various optimization metrics to achieve both diversity and array gains.

2. When the transmitter has no channel knowledge, pre-processing or precoding known as space-time coding is used to achieve a diversity gain but no array gain.

In this paper both of these approaches are combined in one cellular system to achieve full mobility with huge data rates at the best quality of service.

\section{PROPOSED DISTRIBUTED ANTENNA SYSTEM}

In the traditional centralized antenna systems (CAS), antennas of a base station are co-located at the cell center. Mobile station at the cell edge is of high probability working at low signal to noise ratio and high frame error rate because the received power decays with traveling distance by the path-loss in urban environment. As we know the cellular system is consist of multiple cells and the border of they have high probability of interference so boosting transmits power to improve SNR and decrease the frame error rate at the cell edge is not quite cost-efficient because the power of interference is also increased [8]. A general proposed architecture of DAS in multi cell environment is given by the figure No 1 as below'

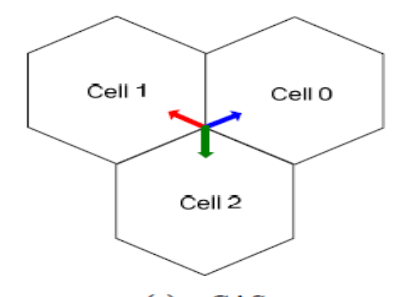

(a) CAS

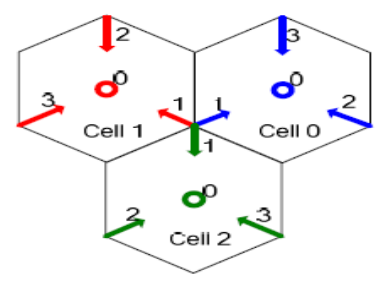

(b) DAS

Figure No 1: Antenna deployment

Where a cell is covered by a small base station and three or six distributed antenna modules. In contrast, the same area is covered by only a single high power base station in the traditional antenna systems. The actual number of distributed antenna modules would be determined by coverage, user densities and other environmental factors but we only consider three distributed antenna modules as a reasonable example [4]. If we expected not to change the shape of the hexagonal sector [8], three directional antennas (denoted by arrows) are placed at the corners and aiming at the Omni-directional antenna (denoted by circle) in the cell center as the figure above [6]. The total transmit power of the $i^{\text {th }}$ distributed antenna module of the $j^{\text {th }}$ cell is $p_{i}^{j}$, where the small base station of each cell are indexed by $\mathrm{i}=0$ and $\mathrm{j}=0$, respectively. Throughout this paper, we assume that the transmitted power of distributed cell is given by the below equation,

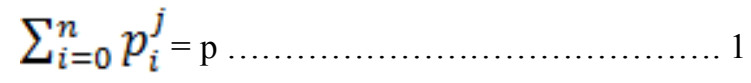

Where $\mathrm{P}$ is the total transmits power of the conventional base station and $\mathrm{n}$ is the number of antennas [4].all the four antennas have the same transmit power [6].

\section{Transmission Strategies of DAS}

In cellular DAS, there are several possible transmissions of downlink strategies using multiple distributed antenna modules. Although many methods of using the distributed antenna modules are possible, we consider two likely transmission strategies of downlink as below points: 
- $\quad$ The blanket transmission scheme or multiple antenna selection (MAS).

- $\quad$ Single transmit selection or single antenna selection (SAS).

The blanket transmission downlink scheme is to transmit signals through all the distributed antenna modules and the home base station due to the less distance between sender and receiver regarding to centralized antenna system (CAS). In this scheme the path loss will be equal to the summation of links from all distributed antennas.

In the single transmit selection scheme, only a single distributed antenna module or the home base station is selected for transmission by the criterion of minimizing propagation path loss. It consider this simple one for simplicity of analysis and since it should minimizing the required transmit power and hence the interference. This scheme is expected to additionally reduce other cell interference (OCI) because the number of OCI sources is reduced [4].

\section{Result \& Discussion}

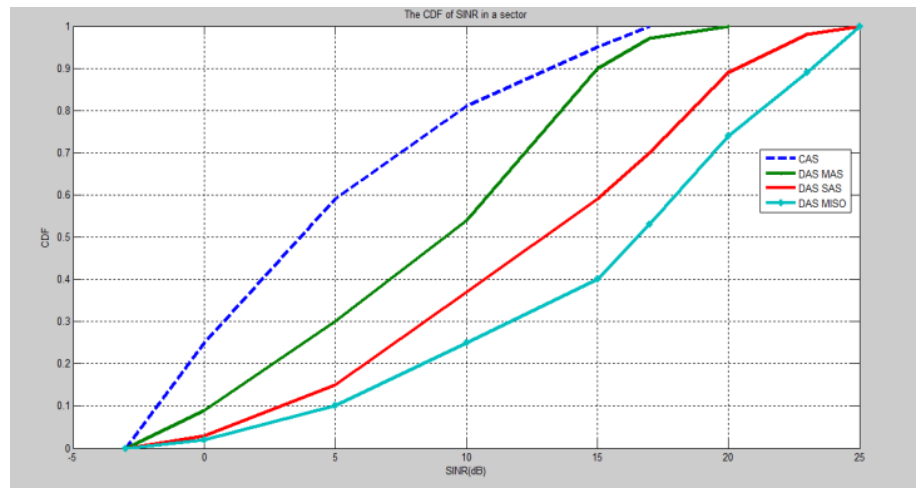

Figure No 2: the CDF of the SINR in a sector

Figure No 2, represents the Cumulative Distribution Function (CDF) of all schemes which are described in the previous sections. Now the comparison is too clear that DAS_MISO is much better than all schemes because the SINR is higher than all schemes and it can accommodate more terminals within the cell.

For example, let us suppose that the minimum value of the received signal $(x)$ is equal to $15 \mathrm{~dB}$ (from the figure No 2), so the probability of all schemes as the below equations;

$$
\begin{aligned}
& \mathrm{CDF}=\mathrm{P}\left(\mathrm{SINR} \_\mathrm{CAS}>15\right)=\mathrm{P}(15<\mathrm{SINR} \text { CAS }<\infty)=F_{\text {SINR }}(\infty)-F_{\text {SINR }}(15)=1-0.95=0.05 \ldots \ldots \ldots 1 \\
& \mathrm{CDF}=\mathrm{P}\left(\mathrm{SINR} \_\mathrm{MAS}>15\right)=\mathrm{P}(15<\mathrm{SINR} \text { MAS }<\infty)=F_{\text {SINR }}(\infty)-F_{\text {SINR }}(15)=1-0.9=0.1 \ldots \ldots \ldots 2 \\
& \mathrm{CDF}=\mathrm{P}\left(\mathrm{SINR} \_\mathrm{SAS}>15\right)=\mathrm{P}\left(15<\mathrm{SINR} \_\mathrm{SAS}<\infty\right)=F_{\text {SINR }}(\infty)-F_{\text {SINR }}(15)=1-0.6=0.4 \ldots \ldots \ldots . .3 \\
& \mathrm{CDF}=\mathrm{P}\left(\mathrm{SINR} \_\mathrm{MISO}>15\right)=\mathrm{P}(15<\mathrm{SINR} \mathrm{MISO}<\infty)=F_{\text {SINR }}(\infty)-F_{\text {SINR }}(15)=1-0.4=0.6 \ldots \ldots \ldots 4
\end{aligned}
$$

\begin{tabular}{|c|c|c|}
\hline \multicolumn{2}{|c|}{ SINR values } \\
\hline Scheme & Probability of receiving SINR & Equation of Probability \\
\hline CAS & 0.05 & 1 \\
\hline DAS_MAS & 0.1 & 2 \\
\hline DAS_SAS & 0.4 & 3 \\
\hline DAS_MISO & 0.6 & 4 \\
\hline
\end{tabular}

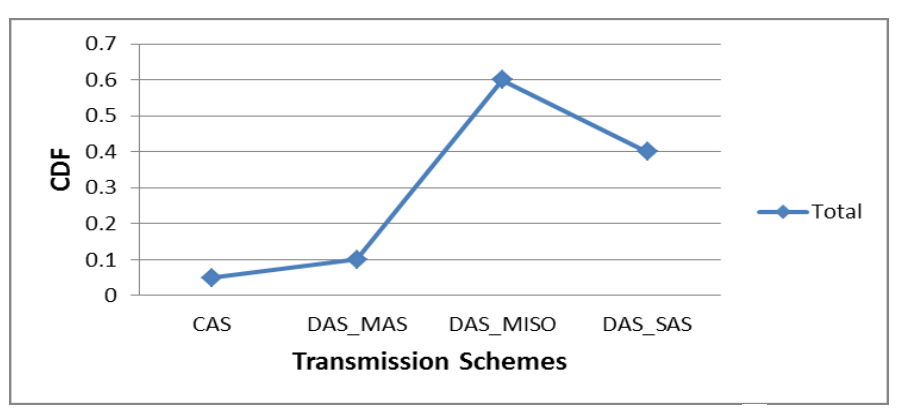

Table and graph No 1: showing the SINR comparison if $\boldsymbol{x}$ equal to $15 \mathrm{~dB}$ 


\section{CONCLUSION}

Radio propagation problems of cellular system have been reviewed and then two approaches of using multiple antennas within the cellular cell have been investigated to overcome the radio propagations problems. Distribution antenna system (DAS) is the first approach of multiple antennas has been showed with its two downlink schemes (DAS_MAS, DAS_SAS). Combining the diversity technique with the DAS is the second approach of using multiple antennas within the cellular cell. The traditional CAS, DAS_SAS, DAS_MAS and DAS MISO are compared and analyzed with their SINR distributions. Simulation results showed that a considerable performance gain can be obtained with a power reduction. Finally, the capacity of the link with multiple antennas configuration have been simulated and proved that it has the biggest capacity due to the multiple antennas configured at its base station.

\section{REFERENCES}

[1] A. Saleh, A. Rustako, and R. Roman. "Distributed antennas for indoor radio communications". IEEE Transactions on Communications. Volume: 35 Issue: 12, pp.1245-1251. December 1987.

[2] K. J. Kerpez. "A radio access system with distributed antennas". IEEE Transactions on Vehicular Technology, Volume: 45, Issue:2, pp.265-275, May 1996.

[3] Jie Zhang and Guillaume de la Roche, "Femtocells: Technologies and Deployment",. 1st ed. England: John Wiley and Sons, 2010.

[4] Wan Choi and J. G. Andrews. "Downlink performance and capacity of distributed antenna systems in a multicell environment". IEEE Transactions on Wireless Communications, Volume: 6, Issue: 1, pp.69- 73, January 2007.

[5] A. Boal, A. Soares, J. C. Silva, and A. Correia. "Distributed antenna cellular system for transmission of broadcast/multicast services”. Vehicular Technology Conference, 2007. VTC2007-Spring. IEEE 65th, pp.1307-1311, April 2007.

[6] Tao Wu, Young Hoon, Jiayin Zhang, Yi Wang, "Distributed Antenna Systems with Power Adjusted Beam Switching", Vehicular Technology Conference. VTC 2010-Spring, 2010 IEEE 71st, pp. 1-5, May 2010.

[7] Jiayin Zhang and Yi Wang, "Energy-Efficient Uplink Transmission in Sectorized Distributed Antenna Systems", 2010 IEEE International Conference on Communications Workshops (ICC), pp.1-5, May 2010.

[8] Jiayin Zhang, Xiaoyan Bi, Yi Wang, “Antenna Pairing for Space- Frequency Block Codes in Edge-Excited Distributed", IEEE PIMRC 2010, pp. 117-122, September 2010.

[9] R1-105600, "One outdoor Scenario for Distributed Antenna for 1.28Mcps TDD”, RAN\#62bis, October 2010.

[10] RP-100178, "Improvements for distributed antenna of 1.28Mcps TDD”, RAN\#47, March 2010.

[11] 3GPP Technical Report, 36.211 V8.9.0, "Physical Channels and Modulation", December 2009.

[12] 3GPP Technical Specification, 36.814 V9.0.0, "Further advancements for E-UTRA physical layer aspects", March 2010.

[13] Saad Al-Hamadi, composite fading channel modeling and information capacity of distribution antenna architectures in cellular networks , doctoral diss., Department of system and computer engineering, Carleton University, Canada, 2010.

[14] Mahanned Mohammed, Enhancement of Mobile radio channel using diversity techniques, M.s thesis, department of electrical and electronic engineering, University of Technology, 2010. 